\title{
Hepatocyte nuclear factor $4 a$ and cancer-related cell signaling pathways: a promising insight into cancer treatment
}

\author{
Duo-Duo Lv', Ling-Yun Zhou' ${ }^{1}$ and Hong Tang ${ }^{1}$
}

\begin{abstract}
Hepatocyte nuclear factor 4a (HNF4a), a member of the nuclear receptor superfamily, is described as a protein that binds to the promoters of specific genes. It controls the expression of functional genes and is also involved in the regulation of numerous cellular processes. A large number of studies have demonstrated that HNF4a is involved in many human malignancies. Abnormal expression of HNF4a is emerging as a critical factor in cancer cell proliferation, apoptosis, invasion, dedifferentiation, and metastasis. In this review, we present emerging insights into the roles of HNF4a in the occurrence, progression, and treatment of cancer; reveal various mechanisms of HNF4a in cancer (e.g., the Wnt/ $\beta$-catenin, nuclear factor-kB, signal transducer and activator of transcription 3 , and transforming growth factor $\beta$ signaling pathways); and highlight potential clinical uses of HNF4a as a biomarker and therapeutic target for cancer.
\end{abstract}

\section{Introduction}

Hepatocyte nuclear factor $4 \alpha$ (HNF4 $\alpha$ ), a member of the nuclear receptor superfamily, is described as a protein that binds to specific DNA sequences and recruits cofactors and the transcription machinery to gene promoters ${ }^{1}$. As one of the key regulators, HNF4 $\alpha$ has been widely associated with a large number of liver-specific genes in various processes, including metabolism, endoderm development and differentiation, and morphogenesis $^{2,3}$. The expression of HNF4 $\alpha$ in the epithelia of digestive and accessory digestive organs ${ }^{4-6}$ suggests that HNF4 $\alpha$ is also important for the specific regulation of gene expression in these tissues ${ }^{2,7}$. Numerous studies have revealed that HNF4 $\alpha$ may play distinct roles in different organ-specific environmental contexts ${ }^{8}$. These distinct roles can be attributed to different HNF4 $\alpha$ isoforms generated by transcription from distinct promoters (P1 and $\mathrm{P} 2)^{7,9}$.

HNF4 $\alpha$ may regulate different signaling pathways by repressing or inducing the expression of downstream

\footnotetext{
Correspondence: Hong Tang (htang6198@hotmail.com)

${ }^{1}$ Center of Infectious Diseases, West China Hospital of Sichuan University,

Chengdu 610041, China
}

target genes to maintain normal physiological activity. Despite the promoter-driven isoforms, dysfunction of HNF4 $\alpha$ clearly triggers the development of distinct diseases $^{8}$. A study found that disruption of HNF4 $\alpha$ causes embryonic lethality with defects in visceral endoderm formation ${ }^{10}$. Conditional knockout of HNF4 $\alpha$ in early liver development damages the development of the hepatic epithelium and liver morphogenesis ${ }^{11}$. Deletion of HNF4 $\alpha$ in the adult liver can result in impaired metabolic homeostasis ${ }^{12}$. In addition to these multiple known functions, HNF4 $\alpha$ has been shown to play an important role in inflammatory processes in internal organs, and accumulating evidence suggests that it is linked to multiple types of cancer $^{13}$.

Recently, additional emerging studies demonstrated that HNF4 $\alpha$ is involved in the proliferation, apoptosis, invasion, and migration of cancer cells both in vitro and in vivo ${ }^{4,14}$. It has been found that HNF4 $\alpha$ has either oncogenic or tumor-suppressive properties in cancer ${ }^{15,16}$. Aberrant expression of HNF4 $\alpha$ is a characteristic of several types of cancer, and altered expression of HNF4 $\alpha$ is strongly associated with the clinical outcome. Moreover, HNF4 $\alpha$ may serve as a novel diagnostic and prognostic

\section{(c) The Author(s) 2021}

(c) (i) Open Access This article is licensed under a Creative Commons Attribution 4.0 International License, which permits use, sharing, adaptation, distribution and reproduction c. in any medium or format, as long as you give appropriate credit to the original author(s) and the source, provide a link to the Creative Commons license, and indicate if changes were made. The images or other third party material in this article are included in the article's Creative Commons license, unless indicated otherwise in a credit line to the material. If material is not included in the article's Creative Commons license and your intended use is not permitted by statutory regulation or exceeds the permitted use, you will need to obtain permission directly from the copyright holder. To view a copy of this license, visit http://creativecommons.org/licenses/by/4.0/. 
biomarker and an effective target for cancer therapy. However, the regulation of HNF4 $\alpha$ in the extracellular and intracellular signaling pathways of tumor pathophysiology is relatively complex, and the underlying tumorigenic or tumor-suppressive functions and potential clinical value of HNF4 $\alpha$ remain elusive. In this review, we focus on the emerging functional role of HNF4 $\alpha$ in a variety of cancers and on the molecular mechanism of $\mathrm{HNF} 4 \alpha$ in the regulation of tumor progression, and we discuss the potential therapeutic uses of HNF4 $\alpha$ in cancer.

\section{Physiological role of HNF4a}

HNF $4 \alpha$ was originally identified in rat liver extracts, binding to sites required for the transcription of transthyretin and apolipoprotein $\mathrm{CIII}^{1}$. Via in situ hybridization analysis, Duncan et al. ${ }^{5}$ found that HNF4 was also expressed in the mesonephric tubules, pancreas, stomach, and intestine and, subsequently, in the metanephric tubules of the developing kidney. Based on this expression pattern, HNF4 was considered to play a role in the earliest stages of murine postimplantation development and organogenesis. Moreover, a study revealed that HNF4 $\alpha$ is expressed at high levels in the liver and kidney and at low levels in $\beta$ cells in the small intestine, colon, and pancreas $^{17}$.

The HNF4 $\alpha$ gene contains 13 exons, spans $>70 \mathrm{~kb}$, and has multiple alternative splice variants. Several splice variants of $H N F 4 \alpha$ are generated by transcription from two alternative promoters (P1 and P2) and by two different ' 3 ' splicing events ${ }^{18}$. It has been proposed that multiple isoforms exist in mammals and that these isoforms are thought to play different physiological roles in the development and transcriptional regulation of target genes $^{7}$. The HNF4 $\alpha$ isoforms driven by the different promoters exhibit tissue-specific expression patterns. Specifically, P1 promoter-driven HNF4 $\alpha$ is expressed in the fetal and adult liver and kidneys, while P2 promoterdriven HNF4 $\alpha$ is expressed in the fetal liver and the adult pancreas and stomach; both isoforms are expressed in the large and small intestines ${ }^{19,20}$. Furthermore, studies have suggested that the HNF4 $\alpha$ isoforms have different activation properties. For instance, in the liver, the expression of HNF4 $\alpha$ is more efficiently initiated from the P2 promoter during early liver development. However, the P1 promoter begins to be favored for transcription of the HNF4 $\alpha$ gene during liver differentiation ${ }^{21}$. Subsequent research suggested that HNF4 $\alpha$ also acts as an oncoprotein that can converge on genes coding for antiapoptotic oncogenes and cytokines and may promote the development of cancer $^{22}$. This apparent paradox could be explained by the existence of two isoform classes produced by transcription from two different promoters.

Recently, HNF4 $\alpha$ has been demonstrated to regulate many important physiological functions of human tissues and organs. For example, HNF4 is required for the development of the liver and can regulate liver functions by controlling the expression of numerous hepaticspecific genes associated with a number of critical metabolic pathways (e.g., glycolysis, gluconeogenesis, fatty acid metabolism, urea production, bile acid synthesis, apolipoprotein synthesis, and drug metabolism $)^{9}$. HNF4 $\alpha$ inactivation experiments in mice clearly demonstrated the important role of this factor in liver differentiation and morphogenesis at different stages of normal development ${ }^{11,23}$. During embryonic colon development and intestinal epithelial cell differentiation, HNF4 $\alpha$ is involved in the control of pancreatic $\beta$-cell proliferation, formation of crypts, maturation of mucin-producing goblet cells, and regulation of the expression of many tissue-specific genes $^{24}$. HNF4 $\alpha$ is differentially expressed in the renal epithelium and can regulate the expression of kidneyspecific genes ${ }^{25}$. Moreover, HNF4 $\alpha$ can activate the expression of multiple genes encoding cell adhesion molecules, extracellular matrix components, cytoskeletal proteins, factors involved in cell survival and proliferation control, and several other $\mathrm{HNFs}^{26}$.

\section{Emerging insights into the roles of HNF4a in cancer}

Although there is an abundance of evidence indicating that HNF4 $\alpha$ plays an important role in embryonic development and controlling biological functions, its role in the regulation of tumorigenesis and cancer development remains unclear. Various studies have documented that aberrant expression of HNF4 $\alpha$ is a potential cancerspecific signature and can be correlated with clinical features in malignant tissues, indicating an important role of HNF4 $\alpha$ in several types of cancer. Collectively, a large body of evidence shows that HNF4 $\alpha$ is associated with the proliferation, differentiation, progression, and metastasis of cancer cells, which could be considered potential prognostic and diagnostic biomarkers during the development of cancer ${ }^{2,24}$. Herein, we discuss emerging insights into the roles of HNF4 $\alpha$ in several types of cancer. Figure 1 summarizes this information.

\section{HNF4a in gastrointestinal cancers Esophageal cancer}

Barrett's metaplasia is an important pathological condition because it is the only known morphological precursor to esophageal adenocarcinoma ${ }^{27}$. Colleypriest et $\mathrm{al}^{28}$ confirmed that HNF4 $\alpha$ is sufficient for the induction of a columnar-like phenotype in the adult mouse esophageal epithelium and is present in Barrett's metaplasia in humans. This observation suggested that induction of HNF4 $\alpha$ is a key early step in the formation of Barrett's metaplasia and is consistent with the origin of Barrett's metaplasia from the esophageal epithelium. 


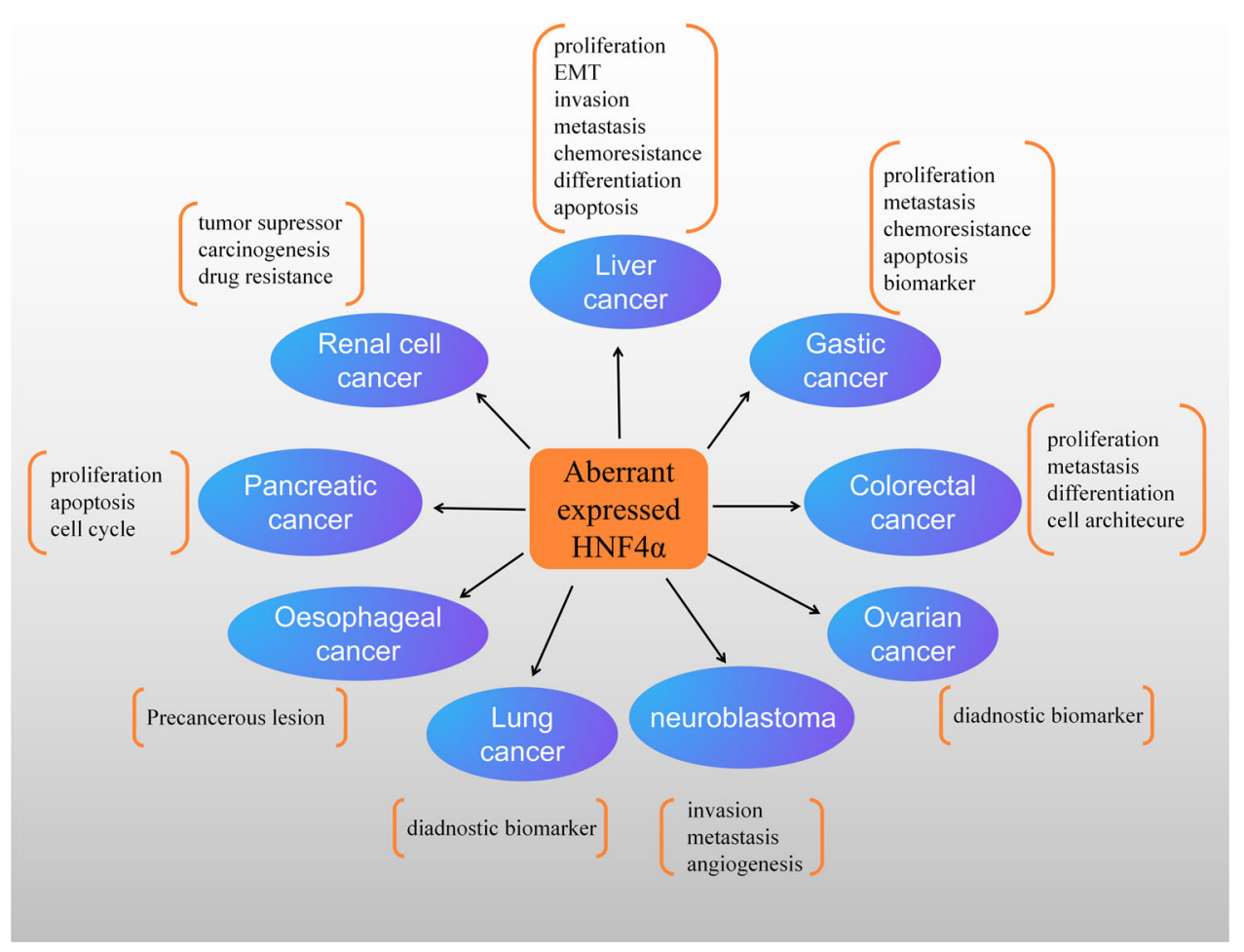

Fig. 1 Biological functions and potential clinical applications of HNF4a in cancer. Abnormal HNF4a expression is observed in a variety of cancer types, including gastrointestinal and digestive cancers, lung cancers, urogenital cancers, and neuroblastoma, which are depicted in the above figure. In cancer, HNF4a can modulate the differentiation, proliferation, apoptosis, invasion, migration, and chemoresistance of cells and may also be used as a diagnostic biomarker.

\section{Gastric cancer (GC)}

$\mathrm{GC}$ is one of the most common causes of cancer-related mortality worldwide. Recent molecular studies have begun to identify the oncogenes and tumor suppressor genes that can directly reprogram the metabolic cycle of GC cells. Notably, HNF4 $\alpha$ is required for cell differentiation and homeostasis in the adult mouse gastric epithelium. However, its deletion causes increased proliferation and collapse of the endoplasmic reticulum and secretory architecture in chief cells in a manner dependent on the HNF4 $\alpha \rightarrow$ X-box binding protein $1 \rightarrow$ MIST1 transcriptional sequence ${ }^{29}$. Recently, it has been shown that overexpression of HNF4 $\alpha$ in GC is essential for GC proliferation in vitro and in vivo ${ }^{30}$. Interestingly, $\mathrm{HNF} 4 \alpha$ acts as an oncogene in GC, and only P2-HNF4 $\alpha$ is expressed in the stomach ${ }^{20,31}$. A functional study analyzing the intestinal phenotype of nonneoplastic and neoplastic gastric gland cells reported that HNF4 $\alpha$ may be involved in the establishment and/or maintenance of the intestinal phenotype of gastric mucosa and adenocarcinoma ${ }^{32}$. Additionally, a study conducted by Xu et al. ${ }^{14}$ highlighted the role of HNF4 $\alpha$ in sustaining oncogenic metabolism in GC cells through the regulation of IDH1. Moreover, Yubo Ma et al. ${ }^{33}$ demonstrated the role of
HNF4 $\alpha$ in chemoresistance in GC, suggesting that HNF4 $\alpha$ may enhance multidrug resistance by regulating apoptosis and the expression of B-cell lymphoma 2 (BCL2). Their results also showed that overexpression of HNF4 $\alpha$ in human GC tissue was associated with more advanced tumor stage and lymph node metastasis. Additionally, HNF4 $\alpha$ has been proposed as a specific biomarker for distinguishing GC tissues from other types of tissues ${ }^{34}$. Consequently, further investigation is warranted to identify the function of HNF4 $\alpha$ and understand the role of HNF4 $\alpha$ in the pathological mechanism of GC and to determine its potential clinical applications.

\section{Colorectal cancer (CRC)}

Previously, studies have found that HNF4 $\alpha$ is involved in the control of intestinal cell proliferation, crypt formation, mucin formation in the regulation of goblet cell maturation, and regulation of the expression of many tissue-specific genes during embryonic colon development and intestinal epithelial cell differentiation ${ }^{24}$. Additionally, it is a key factor in the homeostasis, cell architecture, and barrier function of the adult intestinal epithelium $^{35}$. Recently, the role of HNF4 $\alpha$ in intestinal cancer has been further investigated. As previously 
mentioned, the two promoters are expressed under unique conditions, with the large and small intestines being the only adult tissues that express both P1- and P2HNF4 $\alpha$. Although some studies did not distinguish between the different HNF4 $\alpha$ genes and protein isoforms, several recent studies showed that ectopic expression of P1-HNF4 $\alpha$ but not P2-HNF4 $\alpha$ reduced the tumorigenic potential of HCT116 human colon cancer cells in a mouse xenograft model ${ }^{36,37}$. It was also shown that P1-HNF4 $\alpha$ exerts a differentiative effect on intestinal epithelial cells, while P2-HNF4 $\alpha$ exerts a proliferative effect on these cells $^{36,38}$. Chellappa et al. $^{37}$ observed that lost or mislocalized P1-HNF4 $\alpha$ in $~ 80 \%$ of Dukes stage C colon cancers was correlated with active Src. This finding revealed that Src kinase preferentially phosphorylates P1$\mathrm{HNF} 4 \alpha$ in vitro and in vivo at multiple residues in a complex manner, resulting in loss of function and loss of protein stability of P1-HNF4 $\alpha$ but not P2-HNF4 $\alpha$. These results suggest that different HNF4 $\alpha$ subtypes may actually play different roles in the colon. Furthermore, a study indicated that the increased transcriptional activity of HNF4 $\alpha$ converges on antiapoptotic oncogenes and that cytokines may contribute to the development of $\mathrm{CRC}^{22}$. Thus, considering the unique role of HNF4 $\alpha$ in CRC, targeting HNF4 $\alpha$ may be a promising strategy for the treatment of CRC.

\section{Liver cancer}

Numerous studies have reported that the expression of HNF4 $\alpha$ is dysregulated in hepatocellular carcinoma (HCC) and associated with the development and progression of HCC, thus providing new insight into HCC tumorigenesis $^{26}$. Recent data suggest that HNF4 $\alpha$ is involved in multiple mechanisms and may inhibit the proliferation of hepatocytes. Battle et al. ${ }^{39}$ provided evidence that HNF4 $\alpha$ can regulate the expression of numerous proteins implicated in cell adhesion and junction assembly. As expected, loss of HNF4 $\alpha$ led to the dedifferentiation of hepatocytes. Indeed, accompanied by a decrease in HNF4 $\alpha$ expression, a reduction in cell-cell and cell-extracellular matrix adhesion, loss of cell polarity, an increase in telomerase activity, and inhibition of the expression of liver-specific genes occur in hepatocarcinoma ${ }^{40}$. In addition, it has been reported that HNF4 $\alpha$ is a key control point for the transition to aggressive HCC (from slow-growing to rapidly proliferating $\mathrm{HCC})^{26}$. For example, Yin et al. ${ }^{40}$ demonstrated a striking suppressive effect of HNF4 $\alpha$ on tumorigenesis and tumor development via promotion of cancer stem cell differentiation into mature hepatocytes. This effect led to apoptosis, cell cycle arrest, and cellular senescence. The findings of another study suggested that HNF4 $\alpha$ inhibits the proliferation of hepatocytes by downregulating the expression of oncogenes, such as c-Myc, and shed light on the mechanism underlying HNF $4 \alpha$-mediated inhibition of cell proliferation $^{3}$. Previous studies have linked apoptosis signal-regulating kinase 1 (ASK1) to a variety of cellular functions and pathophysiological processes, such as proliferation, survival, and the inflammatory response ${ }^{41,42}$. Recently, researchers demonstrated that HNF4 $\alpha$ can transcriptionally upregulate ASK1 by directly targeting its promoter in HCC cells. More importantly, strong suppression of ASK1 expression was correlated with decreased HNF4 $\alpha$ levels in HCC tissues, and downregulation of ASK1 partially abrogated the HNF4 $\alpha$ mediated inhibition of $\mathrm{HCC}^{43}$. Furthermore, a recent study conducted by Saha et al. highlighted the importance of HNF4 $\alpha$ in intrahepatic cholangiocarcinoma (IHCC). A genetically engineered mouse model of IHCC expressing mutant isocitrate dehydrogenase (IDH) showed an abnormal response to liver damage in the adult liver; this response was characterized by HNF $4 \alpha$ silencing, impaired differentiation of hepatocytes, and markedly increased cell proliferation. These results revealed a new mechanism in which upregulation of IDH prevents differentiation of liver progenitor cells through inhibition of $\mathrm{HNF}_{4} \alpha^{44}$. Based on this evidence, it can be concluded that HNF4 $\alpha$ may be a key regulator of liver cancer development ${ }^{45}$.

\section{Pancreatic cancer}

The expression of HNF4 $\alpha$ has been found to be aberrant in pancreatic cancer cells. Sun et al. ${ }^{46}$ showed that HNF4 $\alpha$ was upregulated in pancreatic cancer and may be an oncogene. Abrogation of HNF4 $\alpha$ expression inhibited the proliferation of pancreatic cancer cells and induced their apoptosis, with increased expression of the cyclindependent protein kinase inhibitors p21 and p27. In addition, this study demonstrated that increased HNF4 $\alpha$ expression in pancreatic adenocarcinoma was responsible for pancreatic cancer cell proliferation and promoted resistance to gemcitabine by downregulating $\mathrm{hENT}^{46}$. Thus, HNF4 $\alpha$ may serve as a prognostic marker for overall survival, and targeting HNF4 $\alpha$ might reverse gemcitabine resistance and provide novel treatment strategies for pancreatic adenocarcinoma.

\section{Lung cancer}

In some instances, diagnosis of invasive mucinous adenocarcinoma of the lung from a biopsy specimen is difficult because of its minimal nuclear atypia and sparse tumor cells. However, HNF4 (a positive marker) could be useful for identifying invasive mucinous lung adenocarcinoma cells ${ }^{47}$. Furthermore, aiming to clarify the development of a normal counterpart and precancerous lesion of non-terminal respiratory unit (TRU) origin in lung adenocarcinomas, Koji Okudela et al. found that the expression of HNF4 $\alpha$ was similar between bronchiolar metaplastic lesions and terminal bronchioles and that 
some of the metaplastic lesions exhibited an unequivocally higher frequency and expression level of $H N F 4 \alpha$ comparable to that observed in non-TRU lung adenocarcinomas $^{48}$. Therefore, bronchiolar metaplastic lesions strongly expressing $H N F 4 \alpha$ are considered precancerous lesions of non-TRU lung adenocarcinomas.

\section{HNF4a in urogenital cancers \\ Renal cell carcinoma (RCC)}

Sel et al. ${ }^{49}$ was the first to describe altered HNF4 $\alpha$ expression in human RCC by showing its increased expression and DNA binding activity. Subsequently, Lucas et al. ${ }^{25}$ showed based on its downregulation in RCC that HNF4 $\alpha$ played a role as a tumor suppressor. A study revealed that the mRNA levels of $H N F 4 \alpha$ in RCC were downregulated by $4.7-$ fold $^{50}$. Notably, many studies found a strong correlation between the expression of $H N F 4 \alpha$ and E-cadherin in high-grade RCC, which suggests that the regulation of E-cadherin by HNF4 $\alpha$ may be closely associated with the malignancy of $\mathrm{RCC}^{51}$. These results revealed that HNF4 $\alpha$ was downregulated in RCC and that its downregulation was associated with a poor prognosis in patients with RCC. Moreover, inactivation of HNF4 $\alpha$ transcription showed that increased expression and DNA binding activity of $\mathrm{HNF} 4 \alpha$ contribute to carcinogenesis and drug resistance in clear-cell $\mathrm{RCC}^{52}$. Thus, restoration of HNF4 $\alpha$ could render RCC cells more sensitive to chemotherapy. For example, Hagos et al. ${ }^{53}$ showed that HNF4 $\alpha$ increased the expression of organic cation and anion transporters in RCCNG1 cells, thereby increasing the chemosensitivity of tumor cells to oxaliplatin and fluorouracil.

\section{Ovarian cancer}

HNF4 $\alpha$ is expressed in several endodermal tissues. A recent study used a cytological approach to determine that cancer cells in ascites samples from patients with mucinous ovarian adenocarcinoma were HNF4 $\alpha$-positive and that tumor cells in ascites samples from patients with other types of ovarian cancer were HNF4 $\alpha$-negative ${ }^{54}$. Therefore, HNF4 $\alpha$ was revealed to be a useful marker for the histological and cytological diagnosis of ovarian mucinous tumors.

\section{Neuroblastoma}

Neuroblastoma is an extracranial solid tumor that occurs in children and arises from sympathetic neurons via a complex mechanism ${ }^{55}$. A recent analysis of clinical neuroblastoma tissue samples revealed that HNF4 $\alpha$ promoted the invasion, metastasis, and angiogenesis of neuroblastoma cells by targeting matrix metalloproteinase $14^{56}$. Moreover, $\mathrm{Li}$ and $\mathrm{Chen}^{57}$ reported that the overexpression of miR-34a inhibits the proliferation, migration, and invasion of human neuroblastoma SH-SY5Y cells by targeting HNF4 $\alpha$. Additionally, Defeng Deng et al $^{58}$ reported that the long noncoding RNA small nucleolar RNA host gene 16 plays an oncogenic role though the miR-542-3p/HNF4 $\alpha$ axis via the RAS/RAF/ MEK/ERK signaling pathway to induce neuroblastoma growth. These results clarify the functional importance of HNF4 $\alpha$ in neuroblastoma progression.

\section{Signaling pathways of HNF4a in tumor regulation}

In cancer, numerous signaling pathways may have diverse functions and be defined as an interconnected network modulating complex phenomena through a molecular mechanism. Although the major physiological function of signaling pathways is to maintain homeostasis, signaling in normal and oncogenic cells is significantly different. HNF4 $\alpha$ is associated with many signaling pathways that play an important role in tumor transformation, metastasis, inhibition of apoptosis, and promotion of proliferation. Recently, it has been shown that HNF4 $\alpha$ is involved in abnormal activation of one or more signaling pathways (such as the nuclear factor- $\mathrm{kB}(\mathrm{NF}-\mathrm{kB})$ pathway, Wnt/ $\beta$-catenin pathway, and STAT pathway), playing a pivotal role in the occurrence and progression of cancer (Fig. 2).

\section{Wnt/ $\beta$-catenin pathway}

Dysregulation of the Wnt/ $\beta$-catenin signaling pathway is involved in various types of cancer. Researchers previously reported that overexpression of HNF4 $\alpha$ can suppress tumor development through downregulation of the Wnt/ $\beta$-catenin signaling pathway ${ }^{59}$. Overexpression of HNF4 $\alpha$ in cells with a dedifferentiated malignant phenotype restored the cells to an epithelial-like phenotype, indicating that HNF4 $\alpha$ is a regulator of epithelial-mesenchymal transition (EMT). It is well established that EMT is a complex multistep biological process orchestrated by a variety of EMT-inducing transcription factors. This process induces the transdifferentiation of epithelial-like cells into mesenchymal-like cells and facilitates their invasion and migration into blood vessels and lymphatic vessels, thereby participating in the metastasis of a variety of cancers $^{60,61}$. Notably, inhibition of the $\mathrm{Wnt} / \beta$-catenin pathway may downregulate EMT-related markers and decrease cell proliferation and migration ${ }^{62}$. Meng Yang et $\mathrm{al}^{63}$ found that overexpression of HNF4 $\alpha$ completely abolished the Wnt/ $\beta$-catenin signaling-induced EMT phenotype. In particular, HNF4 $\alpha$ inhibits the activation of $\beta$-catenin, which is upstream of SNAIL/SLUG and binds competitively to transcription factor 4 (TCF4) in the nucleus ${ }^{63}$. Conversely, SNAIL inhibits the expression of $\mathrm{HNF} 4 \alpha^{64}$, thereby forming a $\beta$-catenin-SNAIL/SLUGHNF $4 \alpha$ negative feedback circuit. HNF4 $\alpha$ also recruits transcriptional repressors to the promoters of Wnt target genes, further inhibiting the transcription of Wnt/ 


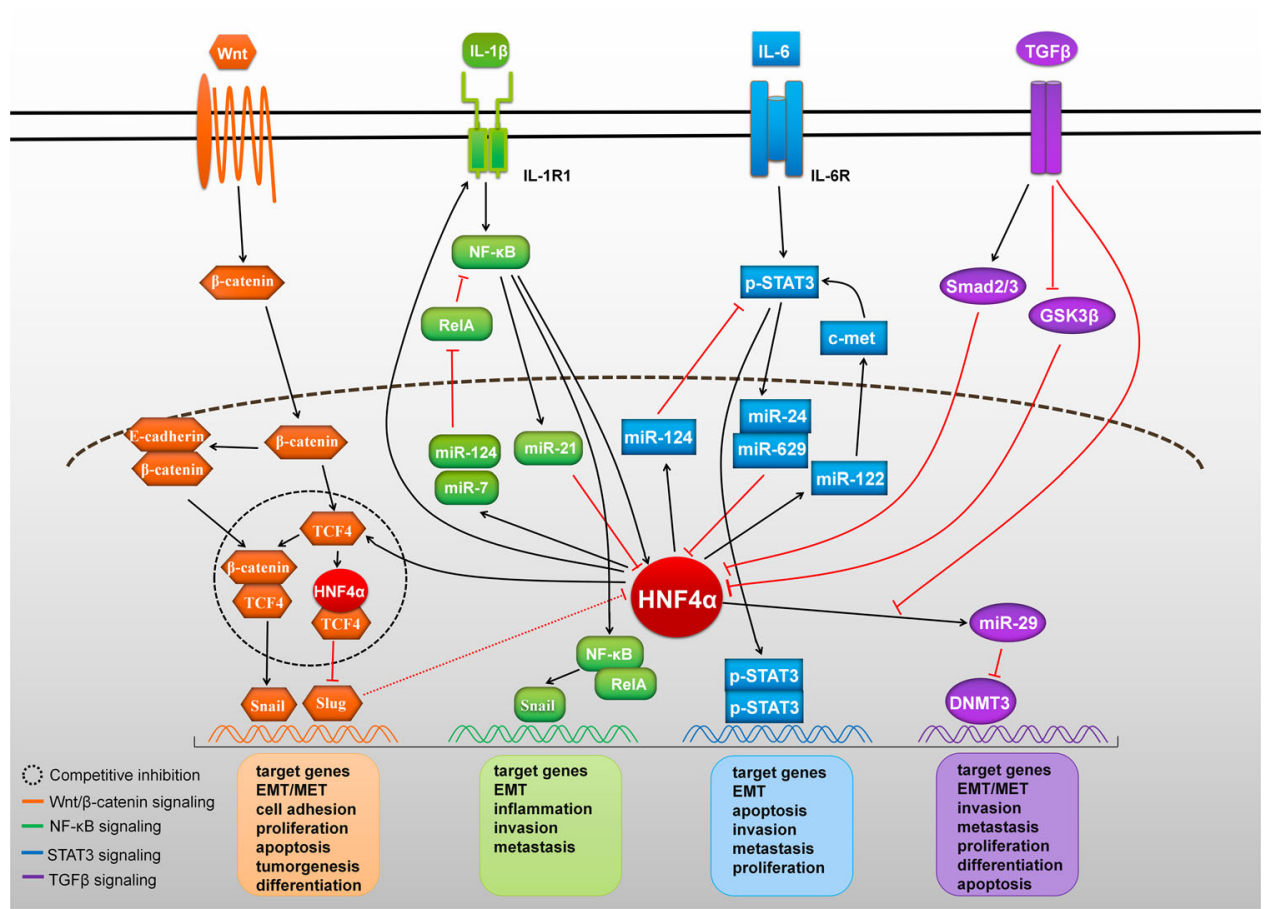

Fig. 2 Role of aberrant HNF4a-related cell signaling pathways in cancer. HNF4a in cancer cells mainly acts through the Wnt/ $\beta$-catenin, NF-KB, STAT3, and TGF $\beta$ signaling pathways to increase cell migration and invasion and decrease apoptosis. High expression of HNF4a regulates target genes by inhibiting the Wnt/ $\beta$-catenin, STAT3 and TGF $\beta$ signaling pathways. After induction by stimuli, high HNF4a expression can activate the NF-KB signaling pathway to promote tumor progression or inhibit the NF-KB signaling pathway to downregulate target genes. Aberrant HNF4a-related Wht/ $\beta$-catenin, NF-KB, STAT3 and TGF $\beta$ signaling pathway activity is involved throughout the EMT process.

$\beta$-catenin signaling pathway target genes (e.g., SLUG and AXIN2 $)^{63}$. In addition, HNF4 $\alpha$ can relocate $\beta$-catenin from the nucleus to the cell membrane, participate in adhesion junctions between epithelial cells, strengthen the epithelial phenotype of cells, reverse the EMT phenotype, and promote the activation of mesenchymal-epithelial transition $(\mathrm{MET})^{65}$. In addition, HNF4 $\alpha$ can directly inhibit the expression of EMT regulatory factors (SNAIL and SLUG) and transform the hepatocyte EMT phenotype into a MET phenotype, thereby inhibiting the progression of cancer ${ }^{66}$. Thus, the double-negative feedback loop formed between Wnt $/ \beta$-catenin signaling and HNF4 $\alpha$ is involved in the regulation of cancer progression.

\section{NF-KB pathway}

Early studies showed that NF- $\mathrm{KB}$ is a central factor in inflammation, cell differentiation and proliferation, and cell death and can be activated by a large variety of stimuli $^{67}$. Recently, NF-kB signaling has been shown to be activated in cancer stem cells, promoting a proinflammatory environment, inhibiting apoptosis, and stimulating cell proliferation ${ }^{68}$. As expected, HNF4 $\alpha$ is involved in the regulation of NF- $\kappa B$ signaling in cancer progression. HNF4 $\alpha$ stimulates the expression of interleukin 1 receptor type 1 (IL1R1) and then amplifies the inflammatory response evoked by its ligand interleukin $1 \beta$ (IL1 $\beta)$. IL1 $\beta / I L 1 R 1$ activates NF- $\mathrm{KB}$ signaling, thereby increasing the expression of HNF4 $\alpha$ and forming a feedback loop that sustains activation of the NF- $\mathrm{kB}$ pathway and drives inflammation toward cancer ${ }^{69}$. In addition, studies have suggested that microRNAs and HNF4 $\alpha$ may cooperate to tune gene expression in distinct biological and pathological processes ${ }^{70}$. Ning et al. $^{71}$ found that HNF4 $\alpha$ directly upregulated the expression of miR-7 and miR-124 in carcinoma cells and downregulated that of the NF- $\mathrm{kB}$ subunit RELA, thereby inhibiting the induction of carcinoma via the NF- $\mathrm{KB}$ signaling pathway. Moreover, $\mathrm{NF}-\mathrm{kB}$ was found to upregulate the expression of miR-21 and inhibit that of HNF4 $\alpha$, thereby forming an HNF4 $\alpha$ NF- $\kappa B$ negative feedback regulatory loop to regulate the course of cancer. Furthermore, NF- $\mathrm{kB}$ promotes and maintains an invasive phenotype of cells and functions as an essential mediator of $\mathrm{EMT}^{72}$. For example, scholars directly introduced the SNAIL gene into a mouse liver cell line and found that it induced EMT accompanied by a decline in the expression of HNF4 $\alpha$. After exogenous introduction of the HNF4 $\alpha$ gene, SNAIL-induced EMT was blocked in liver cells ${ }^{64,73}$. Therefore, in cooperation with microRNAs, HNF4 $\alpha$ inhibits the activation and degradation of SNAIL via NF- $\mathrm{kB}$ by downregulating the 
expression of RELA, thereby blocking the EMT process in tumor cells and alleviating or reversing the pathology of cancer.

\section{HNF4a-signal transducer and activator of transcription 3 (HNF4a-STAT3) pathway}

The link between STAT family proteins and carcinoma in humans is well demonstrated, and constitutively activated STAT3 is crucial for carcinogenesis ${ }^{74}$. STAT3 is considered an oncogene and is highly expressed in a variety of tumor tissues and cells ${ }^{75}$. Sustained activation of STAT3 can cause abnormal proliferation and malignant transformation of tumor cells, enhance the antiapoptotic ability of tumors, and promote tumor invasion, metastasis, and angioplasty ${ }^{76}$. Moreover, the transcriptional program activated by phosphorylated STAT3 in tumors results in the formation of rapidly growing lesions that are highly metastatic ${ }^{77}$. In addition, it has been suggested that phosphorylated STAT3 is positively associated with the expression of the transcription factor TWIST, which is involved in EMT induction, and is negatively correlated with the expression of the epithelial cell marker Ecadherin $^{78}$. E-cadherin is an important factor in invasion and metastasis. Loss of E-cadherin expression stimulates the transformation of cells into a more invasive and less differentiated state through the EMT process ${ }^{79}$. Therefore, activated STAT3 can promote the invasion and metastasis of cancer cells by mediating the EMT process. Hatziapostolou et al. ${ }^{80}$ found that HNF4 $\alpha$ inhibits the activation of STAT3 by directly upregulating the expression of miR-124, thereby blocking the activation of STAT3. They also reported that STAT3 is inhibited by upregulation of miR-24 and miR-629 expression. Expression of HNF4 $\alpha$ forms an HNF4 $\alpha$-STAT3 feedback regulatory loop that regulates the course of carcinoma. Moreover, HNF4 $\alpha$ can cause dysregulation of miR-122 to promote the induction of c-Met and activate the phosphorylation of STAT3, contributing to cancer aggressiveness $^{81}$. Therefore, HNF4 $\alpha$ can alleviate or reverse tumor lesions by blocking the activation of the STAT3 signal transduction pathway and inhibiting the invasion and metastasis of cancer cells.

\section{Transforming growth factor $\beta$ (TGF $\beta$ ) signaling}

The TGF $\beta$ signaling pathway plays important roles in regulating various biological processes, including cell growth, apoptosis, migration, invasion, etc ${ }^{82}$. Previous reports have suggested that TGF $\beta$ signaling plays a dual, opposite role in carcinogenesis. In normal and premalignant cells, it can act as a tumor suppressor. In contrast, during the malignant phases of cancer progression, the TGF $\beta$ signaling pathway triggers tumorpromoting effects, particularly by driving EMT. This event enhances tumor cell migration, invasion, and metastasis to distant organs and ultimately increases resistance to apoptotic stimuli and chemotherapy ${ }^{82,83}$. Interestingly, during postnatal liver development in mice, HNF4 $\alpha$ and TGF $\beta$ are among the first three upstream regulators of gene expression involved ${ }^{84}$. TGF $\beta$ plays a leading role in inhibiting the function of HNF4 $\alpha$ through transcriptional repression and posttranslational modification of HNF4 $\alpha^{85,86}$. TGF $\beta$ inhibits the activity of HNF4 $\alpha$ by targeting this protein for proteasomal degradation in tumor cells ${ }^{85}$. The presence of TGF $\beta$ impairs the efficiency of HNF4 $\alpha$ as a tumor suppressor. Moreover, TGF $\beta$ induces posttranslational modifications of HNF4 $\alpha$, which result in early loss of HNF4 $\alpha$ DNA binding activity toward the target gene promoter ${ }^{86}$. The results of that study also showed that chemical inhibition of glycogen synthase kinase $3 \beta$ (GSK3 $\beta$ ) leads to impairment of HNF4 $\alpha$ binding to DNA. Hence, GSK3 $\beta$ kinase is one of the TGF $\beta$ targets that mediates the inactivation of $\mathrm{HNF} \alpha^{86}$. In addition, HNF4 $\alpha$ exerts epigenetic control of the EMT/MET state in differentiated hepatocytes through miR-29-mediated downregulation of DNA methyltransferases (DNMTs) ${ }^{87,88}$. The degree of miR-29 downregulation and DNMT upregulation is associated with TGF $\beta$-induced EMT and the aggressiveness of cancer ${ }^{89}$. It was further demonstrated that persistent high levels of DNMT maintain DNA methylation, inducing epigenetic changes and participating in EMT and cancer ${ }^{90,91}$. These results reveal that epigenetic regulation of genes by HNF4 $\alpha$ and TGF $\beta$ can be seen as two unique EMT mechanisms in carcinogenesis. Taken together, these results indicate that there is extensive interaction between HNF $4 \alpha$ and TGF $\beta$ during cancer progression.

\section{Therapeutic insights into HNF4a}

Recent evidence suggests that HNF4 $\alpha$ is involved in the proliferation of a variety of cell types throughout the body and can be used as a potential therapeutic target. Nuclear receptors are major therapeutic targets in several metabolic disorders and cancer. This function is largely attributed to their hydrophobic ligand-binding pockets, which are natural targets of small molecules and help regulate the recruitment of coregulators ${ }^{13}$. As a member of the nuclear receptor superfamily of transcription factors, HNF4 $\alpha$ has been reported to possess enormous potential as a clinical therapeutic target in several types of cancer. Yuan et al. ${ }^{92}$ was the first to demonstrate that HNF4 $\alpha$ binds reversibly to the essential fatty acid linoleic acid in mammalian cell culture and mouse liver. This finding suggests the possibility of $\mathrm{HNF} 4 \alpha$ as a drug target. Additional therapeutic drugs can be designed based on the characteristics of $H N F 4 \alpha$, especially for the treatment of cancer. In this context, the pivotal role of HNF4 $\alpha$ as a tumor suppressor indicates the design of promising strategies for the treatment of HCC based on the restoration 
Table 1 A comprehensive list of therapeutic drugs targeting HNF4a activity in cancer.

\begin{tabular}{|c|c|c|c|}
\hline & Cancer type & Mechanism of drug action & References \\
\hline Gene therapy (delivery of LETF) & Liver cancer & $\begin{array}{l}\text { Induces MET and epithelial/hepatic differentiation and blocks EMT } \\
\text { carcinogenesis and metastasis }\end{array}$ & 93 \\
\hline Oroxylin A & Liver cancer & Activates the PKM1/HNF4a pathway & 95 \\
\hline 5-Aza-Cd & Liver cancer & Induces PPARy/RXRa and restores miR-122 expression & 96 \\
\hline Bl6015 & Gastric cancer & Suppresses the Wnt and Notch embryonic signaling pathways & 97 \\
\hline Berberine & Gastric cancer & Is involved in the AMPK-HNF4a-WNT5A signaling pathway & 98 \\
\hline HDAC inhibitors & Colon carcinoma & Downregulates MUC4 & 99 \\
\hline $\begin{array}{l}\text { Dasatinib, AZD-0530 and SKI- } \\
606\end{array}$ & Colorectal cancer & Increases P1-HNF4a protein levels and suppresses colon cancer progression & 13 \\
\hline Oxaliplatin and 5-FU & Renal cell carcinoma & $\begin{array}{l}\text { Overexpression of HNF4a induces chemosensitivity to oxaliplatin and 5-FU } \\
\text { mediated by OCT1 and CNT3 }\end{array}$ & 53 \\
\hline $\begin{array}{l}\text { Apicidin (histone deacetylase } \\
\text { inhibitor) }\end{array}$ & Pancreatic cancer & Reduces the expression of MUC4 and its transcription factor HNF4a & 100 \\
\hline miR-34a & Neuroblastoma & Targets HNF4a to inhibit proliferation, migration and invasion & 57 \\
\hline
\end{tabular}

of HNF4 $\alpha$ expression and function. There is substantial evidence supporting HNF4 $\alpha$ as a "drug" for the treatment of HCC. Marchetti et al. ${ }^{93}$ recently reported the use of members of the liver-enriched transcription factor family, particularly $\mathrm{HNF} 4 \alpha$, as a tool for gene therapy against HCC. As a master regulator of EMT/MET, HNF4 $\alpha$ dynamically restores the differentiation of hepatocytes, induces MET in HCC cells, and controls the epigenetic modification state of differentiated hepatocytes via downregulation of DNA methyltransferases ${ }^{88}$. TGF $\beta$ overrides the tumor-suppressive activity of HNF4 $\alpha$ through the inactivation of GSK3 $\beta$. Future gene therapies against $\mathrm{HCC}$ can be developed based on the inhibition of HNF $4 \alpha$ by TGF $\beta^{86}$. Moreover, from previous research, we have learned that the phosphorylation of paxillin at Tyr118 and autophosphorylation of Src are vital biomarkers of dasatinib activity in tumors for assessing the efficiency of Src activity and tumor growth inhibition ${ }^{94}$. Current Src inhibitors used to treat CRC include dasatinib, AZD-0530, and SKI-606, which are in phase I or phase II clinical trials ${ }^{13}$. Notably, the tyrosine kinase c-Src markedly inhibits the activity of P1-HNF4 $\alpha$ but not that of products of P2-HNF4 $\alpha$ via selective phosphorylation of P1-HNF4 $\alpha$ at tyrosine 23 (Tyr 23) and tyrosine 286 (Tyr 286). This finding indicates that phosphorylation of Tyr 23 and Tyr 286 may be another predictive biomarker for the therapeutic efficacy of Src inhibitors in $\mathrm{CRC}^{37}$. Conversely, previous studies have shown that HNF4 $\alpha$ is specifically overexpressed in GC and is functionally required for the development of GC. Xu et al. ${ }^{14}$ found that the function of HNF4 $\alpha$ in maintaining the oncogenic metabolism of GC cells can be achieved by regulating IDH1.
Therefore, the results of therapeutic studies based on HNF4 $\alpha$ indicate that drug design and development can be performed based on the regulation of HNF4 $\alpha$ in different tumors. In conclusion, HNF4 $\alpha$ has long been recognized as an important regulator of differentiation and is currently associated with cancer. The link between HNF4 $\alpha$ and various cancers can be used to predict the susceptibility of tumors to treatment. While investigations into therapeutic methods based on HNF4 $\alpha$ are currently in early stages, more therapeutic achievements could be attained in the future with an increased understanding of the mechanisms and functions of HNF4 $\alpha$ in cancer. Similarly, the role of different HNF4 $\alpha$ isoforms in cancer is worthy of further study. Therapeutic drugs for different cancers are shown in Table 1.

\section{Conclusion and future perspectives}

In this review, we summarized the molecular mechanisms associated with HNF4 $\alpha$ that regulate multiple processes in cancer. In particular, HNF4 $\alpha$ is abnormally expressed in a cancer-specific manner in various types of tumors and has opposite functions in tumor inhibition and promotion. Overexpression of HNF4 $\alpha$ in different types of tumor cells (e.g., HCC, CRC, and RCC cells) is recognized as a major antitumor factor in suppressing EMT, disease progression, and metastasis; however, it exerts an opposite effect in GC, lung cancer, pancreatic cancer, and neuroblastoma. Therefore, further understanding of the regulatory mechanisms of HNF4 $\alpha$ in different cell types in patients with cancer has the potential to improve the antitumor efficacy of targeting HNF4 $\alpha$ and/or to overcome chemoresistance. Although 
numerous studies have demonstrated that HNF4 $\alpha$ is dysregulated in cancers and may serve as a novel diagnostic biomarker and therapeutic target in cancers, clinical application of HNF4 $\alpha$ remains challenging. Moreover, drugs that target HNF4 $\alpha$ (identified in mechanistic studies) have the potential to increase these benefits when used in combination with other chemotherapeutic drugs to treat tumors.

\section{Acknowledgements}

This work was supported by the National Natural Science Foundation of China (No. 81772193 and 81802468), the 1.3 .5 project for disciplines of excellence, West China Hospital, Sichuan University (No. ZYGD20009 and ZYJC18008), the Sichuan Province Key Technologies R\&D Program (19ZDYF), the Sichuan Science and Technology Program 2019YFS0207, and the China Postdoctoral Science Foundation 2020M670062ZX to Dr. Lingyun Zhou.

\section{Conflict of interest}

The authors declare that they have no conflict of interest.

\section{Publisher's note}

Springer Nature remains neutral with regard to jurisdictional claims in published maps and institutional affiliations.

Received: 26 March 2020 Revised: 23 October 2020 Accepted: 19 November 2020.

Published online: 18 January 2021

\section{References}

1. Sladek, F. M., Zhong, W. M., Lai, E. \& Darnell, J. E. Jr. Liver-enriched transcription factor HNF-4 is a novel member of the steroid hormone receptor superfamily. Genes Dev. 4, 2353-2365 (1990).

2. Yamagata, K et al. Mutations in the hepatocyte nuclear factor-4alpha gene in maturity-onset diabetes of the young (MODY1). Nature 384, 458-460 (1996).

3. Walesky, C. et al. Hepatocyte nuclear factor 4 alpha deletion promotes diethylnitrosamine-induced hepatocellular carcinoma in rodents. Hepatology 57, 2480-2490 (2013)

4. Babeu, J. P., Darsigny, M., Lussier, C. R. \& Boudreau, F. Hepatocyte nuclear factor 4alpha contributes to an intestinal epithelial phenotype in vitro and plays a partial role in mouse intestinal epithelium differentiation. Am. J. Physiol. Gastrointest. Liver Physiol. 297, G124-G134 (2009).

5. Duncan, S. A. et al. Expression of transcription factor HNF-4 in the extraembryonic endoderm, gut, and nephrogenic tissue of the developing mouse embryo: HNF-4 is a marker for primary endoderm in the implanting blastocyst. Proc. Natl Acad. Sci. USA 91, 7598-7602 (1994).

6. Taraviras, S., Monaghan, A. P., Schutz, G. \& Kelsey, G. Characterization of the mouse HNF-4 gene and its expression during mouse embryogenesis. Mechanisms Dev. 48, 67-79 (1994)

7. Jiang, S. et al. Expression and localization of P1 promoter-driven hepatocyte nuclear factor-4alpha (HNF4alpha) isoforms in human and rats. Nucl. receptor 1, 5 (2003).

8. Babeu, J. P. \& Boudreau, F. Hepatocyte nuclear factor 4-alpha involvement in liver and intestinal inflammatory networks. World J. Gastroenterol. 20, 22-30 (2014).

9. Lu, H. Crosstalk of HNF4alpha with extracellular and intracellular signaling pathways in the regulation of hepatic metabolism of drugs and lipids. Acta Pharm. Sin. B 6, 393-408 (2016).

10. Chen, W. S. et al. Disruption of the HNF-4 gene, expressed in visceral endoderm, leads to cell death in embryonic ectoderm and impaired gastrulation of mouse embryos. Genes Dev. 8, 2466-2477 (1994).

11. Parviz, F. et al. Hepatocyte nuclear factor 4 alpha controls the development of a hepatic epithelium and liver morphogenesis. Nat. Genet. 34, 292-296 (2003).

12. Hayhurst, G. P., Lee, Y. H., Lambert, G., Ward, J. M. \& Gonzalez, F. J. Hepatocyte nuclear factor 4 alpha (nuclear receptor 2A1) is essential for maintenance of hepatic gene expression and lipid homeostasis. Mol. Cell. Biol. 21, 1393-1403 (2001).

13. Chellappa, K., Robertson, G. R. \& Sladek, F. M. HNF4alpha: a new biomarker in colon cancer? Biomark. Med. 6, 297-300 (2012)

14. $\mathrm{Xu}, \mathrm{C}$. et al. HNF4alpha pathway mapping identifies wild-type $\mathrm{IDH} 1$ as a targetable metabolic node in gastric cancer. Gut https://doi.org/10.1136/ gutjnl-2018-318025 (2019).

15. Chellappa, K. et al. Opposing roles of nuclear receptor HNF4alpha isoforms in colitis and colitis-associated colon cancer. Elife https://doi.org/10.7554/ elife.10903 (2016).

16. Tsai, P. H. et al. Dual delivery of HNF4alpha and cisplatin by mesoporous silica nanoparticles inhibits cancer pluripotency and tumorigenicity in hepatomaderived CD133-expressing stem cells. ACS Appl Mater. Interfaces 11, 19808-19818 (2019).

17. Walesky, C. \& Apte, U. Role of hepatocyte nuclear factor 4alpha (HNF4alpha) in cell proliferation and cancer. Gene Expr. 16, 101-108 (2015).

18. Yusuf, D. et al. The transcription factor encyclopedia. Genome Biol. 13, R24 (2012).

19. Tanaka, T. et al. Dysregulated expression of P1 and P2 promoter-driven hepatocyte nuclear factor-4alpha in the pathogenesis of human cancer. $J$. Pathol. 208, 662-672 (2006).

20. Dean, S., Tang, J. I., Seckl, J. R. \& Nyirenda, M. J. Developmental and tissuespecific regulation of hepatocyte nuclear factor 4-alpha (HNF4-alpha) isoforms in rodents. Gene Expr. 14, 337-344 (2010).

21. Torres-Padilla, M. E., Fougere-Deschatrette, C. \& Weiss, M. C. Expression of HNF4alpha isoforms in mouse liver development is regulated by sequential promoter usage and constitutive $3^{\prime}$ end splicing. Mechanisms Dev. 109, 183-193 (2001).

22. Schwartz, B. et al. Inhibition of colorectal cancer by targeting hepatocyte nuclear factor-4alpha. Int. J. Cancer 124, 1081-1089 (2009).

23. Li, J., Ning, G. \& Duncan, S. A. Mammalian hepatocyte differentiation requires the transcription factor HNF-4alpha. Genes Dev. 14, 464-474 (2000).

24. Garrison, W. D. et al. Hepatocyte nuclear factor 4alpha is essential for embryonic development of the mouse colon. Gastroenterology $\mathbf{1 3 0}$ 1207-1220 (2006).

25. Lucas, B. et al. HNF4alpha reduces proliferation of kidney cells and affects genes deregulated in renal cell carcinoma. Oncogene 24, 6418-6431 (2005)

26. Lazarevich, N. L. et al. Deregulation of hepatocyte nuclear factor 4 (HNF4)as a marker of epithelial tumors progression. Exp. Oncol. 32, 167-171 (2010).

27. Fitzgerald, R. C. Molecular basis of Barrett's oesophagus and oesophageal adenocarcinoma. Gut 55, 1810-1820 (2006).

28. Colleypriest, B. J. et al. Hnf4alpha is a key gene that can generate columnar metaplasia in oesophageal epithelium. Differentiation 93, 39-49 (2017).

29. Moore, B. D., Khurana, S. S., Huh, W. J. \& Mills, J. C. Hepatocyte nuclear factor 4alpha is required for cell differentiation and homeostasis in the adult mouse gastric epithelium. Am. J. Physiol. Gastrointest. Liver Physiol. 311, G267-G275 (2016).

30. Chia, N. Y. et al. Regulatory crosstalk between lineage-survival oncogenes KLF5, GATA4 and GATA6 cooperatively promotes gastric cancer development. Gut 64, 707-719 (2015).

31. Chang, H. R. et al. HNF4alpha is a therapeutic target that links AMPK to WNT signalling in early-stage gastric cancer. Gut 65, 19-32 (2016).

32. Kojima, $K$ et al. The expression of hepatocyte nuclear factor-4alpha, a developmental regulator of visceral endoderm, correlates with the intestinal phenotype of gastric adenocarcinomas. Pathology 38, 548-554 (2006).

33. $\mathrm{Ma}, \mathrm{Y} ., \mathrm{Wei}, \mathrm{X} . \& \mathrm{Wu}, \mathrm{Z}$. HNF-4alpha promotes multidrug resistance of gastric cancer cells through the modulation of cell apoptosis. Oncol. Lett. 14, 6477-6484 (2017).

34. van der Post, R. S. et al. HNF4A immunohistochemistry facilitates distinction between primary and metastatic breast and gastric carcinoma. Virchows Arch 464, 673-679 (2014).

35. Cattin, A. L. et al. Hepatocyte nuclear factor 4 alpha, a key factor for homeostasis, cell architecture, and barrier function of the adult intestinal epithelium. Mol. Cell. Biol. 29, 6294-6308 (2009).

36. Vuong, L. M. et al. Differential effects of hepatocyte nuclear factor 4alpha isoforms on tumor growth and t-cell factor 4/AP-1 interactions in human colorectal Cancer cells. Mol. Cell. Biol. 35, 3471-3490 (2015).

37. Chellappa, K. et al. Src tyrosine kinase phosphorylation of nuclear receptor HNF4alpha correlates with isoform-specific loss of HNF4alpha in human colon cancer. Proc. Natl Acad. Sci. USA 109, 2302-2307 (2012). 
38. Babeu, J. P., Jones, C., Geha, S., Carrier, J. C. \& Boudreau, F. P1 promoter-driven HNF4alpha isoforms are specifically repressed by beta-catenin signaling in colorectal cancer cells. J. Cell Sci. https://doi.org/10.1242/jcs.214734 (2018).

39. Battle, M. A. et al. Hepatocyte nuclear factor 4alpha orchestrates expression of cell adhesion proteins during the epithelial transformation of the developing liver. Proc. Natl Acad. Sci. USA 103, 8419-8424 (2006).

40. Yin, C. et al. Differentiation therapy of hepatocellular carcinoma in mice with recombinant adenovirus carrying hepatocyte nuclear factor-4alpha gene. Hepatology 48, 1528-1539 (2008).

41. Ichijo, H. et al. Induction of apoptosis by ASK1, a mammalian MAPKKK that activates SAPKJJNK and p38 signaling pathways. Science 275, 90-94 (1997).

42. Matsuzawa, A. et al. ROS-dependent activation of the TRAF6-ASK1-p38 pathway is selectively required for TLR4-mediated innate immunity. Nat. Immunol. 6, 587-592 (2005)

43. Jiang, C. F. et al. Apoptosis signal-regulating kinase 1 mediates the inhibitory effect of hepatocyte nuclear factor-4alpha on hepatocellular carcinoma. Oncotarget 7, 27408-27421 (2016).

44. Saha, S. K. et al. Mutant IDH inhibits HNF-4alpha to block hepatocyte differentiation and promote biliary cancer. Nature 513, 110-114 (2014).

45. Yan, $\mathrm{H}$. et al. Identification of potential transcription factors, long noncoding RNAs, and microRNAs associated with hepatocellular carcinoma. J. Cancer Res. Ther. 14, S622-S627 (2018).

46. Sun, Q. et al. Role of hepatocyte nuclear factor 4 alpha in cell proliferation and gemcitabine resistance in pancreatic adenocarcinoma. Cancer Cell Int 19, 49 (2019).

47. Sugano, $M$. et al. HNF4alpha as a marker for invasive mucinous adenocarcinoma of the lung. Am. J. Surg. Pathol. 37, 211-218 (2013).

48. Okudela, K. et al. A subpopulation of airway epithelial cells that express hepatocyte nuclear factor 4alpha - its implication in the development of non-terminal respiratory unit-type lung adenocarcinoma. Histol. Histopathol. 34, 1217-1227 (2019)

49. Sel, S., Ebert, T., Ryffel, G. U. \& Drewes, T. Human renal cell carcinogenesis is accompanied by a coordinate loss of the tissue specific transcription factors HNF4 alpha and HNF1 alpha. Cancer Lett. 101, 205-210 (1996).

50. Wirsing, A., Senkel, S., Klein-Hitpass, L. \& Ryffel, G. U. A systematic analysis of the $3^{\prime} U T R$ of HNF4A mRNA reveals an interplay of regulatory elements including miRNA target sites. PLOS ONE 6, e27438 (2011).

51. Gao, Y. et al. HNF4alpha downregulation promotes tumor migration and invasion by regulating Ecadherin in renal cell carcinoma. Oncol. Rep. 42, 1066-1074 (2019).

52. Gao, Y. H. et al. VHL deficiency augments anthracycline sensitivity of clear cel renal cell carcinomas by down-regulating ALDH2. Nat. Commun. 8, 15337 (2017)

53. Hagos, Y. et al. HNF4alpha induced chemosensitivity to oxaliplatin and 5-FU mediated by OCT1 and CNT3 in renal cell carcinoma. J. Pharm. Sci. 103, 3326-3334 (2014).

54. Sugai, M. et al. Expression of hepatocyte nuclear factor 4 alpha in primary ovarian mucinous tumors. Pathol. Int. 58, 681-686 (2008).

55. Al-Shammari, N. F., Redha, E. \& Al Hajeri, M. H. Cervical neonatal neuroblastoma with recurrent SVT. Gulf J. Oncol. 45-57 (2009).

56. Xiang, $X$. et al. Hepatocyte nuclear factor 4 alpha promotes the invasion, metastasis and angiogenesis of neuroblastoma cells via targeting matrix metalloproteinase 14. Cancer Lett. 359, 187-197 (2015).

57. Li, Z. \& Chen, H. miR-34a inhibits proliferation, migration and invasion of paediatric neuroblastoma cells via targeting HNF4alpha. Artif. Cells Nanomed. Biotechnol. 47, 3072-3078 (2019).

58. Deng, D., Yang, S. \& Wang, X. Long non-coding RNA SNHG16 regulates cell behaviors through miR-542-3p/HNF4alpha axis via RAS/RAF/MEK/ERK signaling pathway in pediatric neuroblastoma cells. Biosci. Rep. https://doi.org/ 10.1042/BSR20200723 (2020).

59. Wu, N. et al. Overexpression of hepatocyte nuclear factor 4alpha in human mesenchymal stem cells suppresses hepatocellular carcinoma development through Wnt/beta-catenin signaling pathway downregulation. Cancer Biol. Ther. 17, 558-565 (2016).

60. Thiery, J. P., Acloque, H., Huang, R. Y. \& Nieto, M. A. Epithelial-mesenchymal transitions in development and disease. Cell 139, 871-890 (2009).

61. Nieto, M. A. Epithelial-mesenchymal transitions in development and disease: old views and new perspectives. Int. J. Dev. Biol. 53, 1541-1547 (2009).

62. Huang, Q. et al. Tg737 regulates epithelial-mesenchymal transition and cancer stem cell properties via a negative feedback circuit between Snail and
HNF4alpha during liver stem cell malignant transformation. Cancer Lett. 402 52-60 (2017).

63. Yang, M. et al. A double-negative feedback loop between Wnt-beta-catenin signaling and HNF4alpha regulates epithelial-mesenchymal transition in hepatocellular carcinoma. J. Cell Sci. 126, 5692-5703 (2013).

64. Cicchini, C. et al. Snail controls differentiation of hepatocytes by repressing HNF4alpha expression. J. Cell Physiol. 209, 230-238 (2006).

65. Ning, B. F. et al. Hepatocyte nuclear factor 4 alpha suppresses the development of hepatocellular carcinoma. Cancer Res. 70, 7640-7651 (2010).

66. Yao, D., Peng, S. \& Dai, C. The role of hepatocyte nuclear factor 4alpha in metastatic tumor formation of hepatocellular carcinoma and its close relationship with the mesenchymal-epithelial transition markers. BMC Cancer $\mathbf{1 3}$ 432 (2013).

67. Hoesel, B. \& Schmid, J. A. The complexity of NF-kappaB signaling in inflammation and cancer. Mol. Cancer 12, 86 (2013).

68. Chefetz, I., Holmberg, J. C., Alvero, A. B., Visintin, I. \& Mor, G. Inhibition of Aurora-A kinase induces cell cycle arrest in epithelial ovarian cancer stem cells by affecting NFkB pathway. Cell Cycle 10, 2206-2214 (2011).

69. Ma, L. et al. Mutual amplification of HNF4alpha and IL-1R1 composes an inflammatory circuit in Helicobacter pylori associated gastric carcinogenesis. Oncotarget 7, 11349-11363 (2016).

70. Morimoto, A. et al. An HNF4alpha-microRNA-194/192 signaling axis maintains hepatic cell function. J. Biol. Chem. 292, 10574-10585 (2017).

71. Ning, B. F. et al. Hepatocyte nuclear factor 4alpha-nuclear factor-kappaB feedback circuit modulates liver cancer progression. Hepatology $\mathbf{6 0}$ 1607-1619 (2014)

72. Huang, T., Chen, Z. \& Fang, L. Curcumin inhibits LPS-induced EMT through downregulation of NF-kappaB-Snail signaling in breast cancer cells. Oncol. Rep. 29, 117-124 (2013).

73. Tsubaki, M. et al. Activation of NF-kappaB by the RANKL/RANK system up-regulates snail and twist expressions and induces epithelial-tomesenchymal transition in mammary tumor cell lines. J. Exp. Clin Cancer Res. 32, 62 (2013).

74. Grandis, J. R. et al. Requirement of Stat3 but not Stat1 activation for epidermal growth factor receptor- mediated cell growth In vitro. J. Clin. Invest. 102, 1385-1392 (1998)

75. Avalle, L., Pensa, S., Regis, G., Novelli, F. \& Poli, V. STAT1 and STAT3 in tumorigenesis: a matter of balance. JAKSTAT 1, 65-72 (2012).

76. Loh, C. Y. et al. Signal transducer and activator of transcription (STATs) proteins in cancer and inflammation: functions and therapeutic implication Front. Oncol. 9, 48 (2019).

77. Haricharan, S. \& Li, Y. STAT signaling in mammary gland differentiation, cell survival and tumorigenesis. Mol. Cell Endocrinol. 382, 560-569 (2014).

78. Yang, J. et al. Twist, a master regulator of morphogenesis, plays an essential role in tumor metastasis. Cell 117, 927-939 (2004).

79. Bure, I. V., Nemtsova, M. V. \& Zaletaev, D. V. Roles of E-cadherin and noncoding RNAs in the epithelial-mesenchymal transition and progression in gastric cancer. Int. J. Mol. Sci. https://doi.org/10.3390/ijms20122870 (2019).

80. Hatziapostolou, M. et al. An HNF4alpha-miRNA inflammatory feedback circuit regulates hepatocellular oncogenesis. Cell 147, 1233-1247 (2011).

81. Yang, Y. M. et al. Galpha12 overexpressed in hepatocellular carcinoma reduces microRNA-122 expression via HNF4alpha inactivation, which causes c-Met induction. Oncotarget 6, 19055-19069 (2015).

82. Panda, M. \& Biswal, B. K. Cell signaling and cancer: a mechanistic insight into drug resistance. Mol. Biol. Rep. https://doi.org/10.1007/s11033-019-04958-6 (2019).

83. Suriyamurthy, S., Baker, D., Ten Dijke, P. \& Iyengar, P. V. Epigenetic reprogramming of TGF-beta signaling in breast cancer. Cancers (Basel), https://doi. org/10.3390/cancers11050726 (2019).

84. Gunewardena, S. S. et al. Deciphering the developmental dynamics of the mouse liver transcriptome. PLOS ONE 10, e0141220 (2015).

85. de Lucas, S., Lopez-Alcorocho, J. M., Bartolome, J. \& Carreno, V. Nitric oxide and TGF-beta1 inhibit HNF-4alpha function in HEPG2 cells. Biochem. Biophys. Res. Commun. 321, 688-694 (2004).

86. Cozzolino, A. M. et al. TGFbeta overrides HNF4alpha tumor suppressing activity through GSK3beta inactivation: implication for hepatocellular carcinoma gene therapy. J. Hepatol. 58, 65-72 (2013).

87. Santangelo, L. et al. The stable repression of mesenchymal program is required for hepatocyte identity: a novel role for hepatocyte nuclear factor 4alpha. Hepatology 53, 2063-2074 (2011). 
88. Cicchini, $C$. et al. Epigenetic control of EMT/MET dynamics: HNF4alpha impacts DNMT3s through miRs-29. Biochim. Biophys. Acta 1849, 919-929 (2015).

89. Kogure, T. et al. Involvement of miRNA-29a in epigenetic regulation of transforming growth factor-beta-induced epithelial-mesenchymal transition in hepatocellular carcinoma. Hepatol. Res. 44, 907-919 (2014).

90. Carmona, F. J. et al. A comprehensive DNA methylation profile of epithelialto-mesenchymal transition. Cancer Res. 74, 5608-5619 (2014).

91. Saito, Y. et al. Increased protein expression of DNA methyltransferase (DNMT) 1 is significantly correlated with the malignant potential and poor prognosis of human hepatocellular carcinomas. Int. J. Cancer 105, 527-532 (2003).

92. Yuan, $X$. et al. Identification of an endogenous ligand bound to a native orphan nuclear receptor. PLOS ONE 4, e5609 (2009).

93. Marchetti, A., Bisceglia, F., Cozzolino, A. M. \& Tripodi, M. New Tools for Molecular Therapy of Hepatocellular Carcinoma. Diseases 3, 325-340 (2015).

94. Serrels, A. et al. Identification of potential biomarkers for measuring inhibition of Src kinase activity in colon cancer cells following treatment with dasatinib. Mol. Cancer Ther. 5, 3014-3022 (2006).
95. Wei, L. et al. Oroxylin A activates PKM1/HNF4 alpha to induce hepatoma differentiation and block cancer progression. Cell Death Dis. 8 , e2944 (2017)

96. Song, $K$. et al. Epigenetic regulation of MicroRNA-122 by peroxisome proliferator activated receptor-gamma and hepatitis $b$ virus $X$ protein in hepatocellular carcinoma cells. Hepatology 58, 1681-1692 (2013).

97. Kim, J. H. et al. Differential effects, on oncogenic pathway signalling, by derivatives of the HNF4 alpha inhibitor Bl6015. Br. J. Cancer 120, 488-498 (2019).

98. Hu, Q., Li, L., Zou, X., Xu, L. \& Yi, P. Berberine attenuated proliferation, invasion and migration by targeting the AMPK/HNF4alpha/WNT5A pathway in gastric carcinoma. Front Pharm. 9, 1150 (2018).

99. Algamas-Dimantov, A., Yehuda-Shnaidman, E., Peri, I. \& Schwartz, B. Epigenetic control of HNF-4alpha in colon carcinoma cells affects MUC4 expression and malignancy. Cell Oncol. (Dordr.) 36, 155-167 (2013).

100. Ansari, D. et al. Apicidin sensitizes pancreatic cancer cells to gemcitabine by epigenetically regulating MUC4 expression. Anticancer Res. 34, 5269-5276 (2014). 